\title{
On the nonlinear stabilization of the wave equation
}

\author{
by Aissa Guesmia (Strasbourg)
}

\begin{abstract}
We obtain a precise decay estimate of the energy of the solutions to the initial boundary value problem for the wave equation with nonlinear internal and boundary feedbacks. We show that a judicious choice of the feedbacks leads to fast energy decay.
\end{abstract}

1. Introduction. Let $\Omega$ be a bounded open set in $\mathbb{R}^{n}(n=1,2, \ldots)$ having a boundary $\Gamma$ of class $C^{2}$. We denote by $\nu$ the outward unit normal vector to $\Gamma$. Fix a point $x_{0} \in \mathbb{R}^{n}$, and set

$$
m(x):=x-x_{0}, \quad x \in \mathbb{R}^{n}, \quad \text { and } \quad R=\|m\|_{L^{\infty}(\Omega)} .
$$

Let $\Gamma_{0}$ be an open subset of $\Gamma$ such that setting $\Gamma_{1}=\Gamma \backslash \Gamma_{0}$ we have

$$
m . \nu>0 \quad \text { on } \Gamma_{1}, \quad m . \nu \leq 0 \text { on } \Gamma_{0} .
$$

Let $f, g: \mathbb{R} \rightarrow \mathbb{R}$ be two nondecreasing continuous functions such that $f(0)=g(0)=0$, and let $a$ be a nonnegative number for simplicity. (It is easy to generalize our results to the case where $a$ is a nonnegative function in $C\left(\bar{\Gamma}_{1}\right)$.) Consider the following system:

$$
\begin{gathered}
u^{\prime \prime}-\Delta u+f\left(u^{\prime}\right)=0 \quad \text { in } \Omega \times \mathbb{R}^{+}, \\
u=0 \quad \text { on } \Gamma_{0} \times \mathbb{R}^{+}, \\
\partial_{\nu} u+(m . \nu)\left(a u+g\left(u^{\prime}\right)\right)=0 \quad \text { on } \Gamma_{1} \times \mathbb{R}^{+}, \\
u(0)=u_{0} \quad \text { and } \quad u^{\prime}(0)=u_{1} \quad \text { on } \Omega
\end{gathered}
$$

where we use the notation $\mathbb{R}^{+}:=[0, \infty)$. Define the energy of the solution by the formula

$$
E(t):=\frac{1}{2} \int_{\Omega}\left(\left(u^{\prime}\right)^{2}+|\nabla u|^{2}\right) d x+\frac{a}{2} \int_{\Gamma_{1}}(m . \nu) u^{2} d \Gamma, \quad t \in \mathbb{R}^{+} .
$$

1991 Mathematics Subject Classification: 35L05, 93D15, 35B40.

Key words and phrases: wave equation, nonlinear damping, integral inequality. 
An easy formal computation shows that

$$
E^{\prime}(t)=-\int_{\Omega} u^{\prime} f\left(u^{\prime}\right) d x-\int_{\Gamma_{1}}(m . \nu) u^{\prime} g\left(u^{\prime}\right) d \Gamma
$$

(observe that $x f(x), x g(x) \geq 0$ for every $x \in \mathbb{R}$ ); hence the energy is nonincreasing.

This system is well-posed in the following sense (cf. [1, 2, 4] for second order evolution equations and $[5,6,9,10,11]$ for the wave equation with a nonlinear internal or boundary feedback): let us introduce three real Hilbert spaces $H, V$ and $W$ by setting

$$
H=L^{2}(\Omega), \quad V=H_{\Gamma_{0}}^{1}(\Omega) \quad \text { and } \quad W=H^{2}(\Omega) \cap V
$$

where $H_{\Gamma_{0}}^{1}(\Omega)=\left\{v \in H^{1}(\Omega): v=0\right.$ on $\left.\Gamma_{0}\right\}$ and assume that there exists a positive constant $c^{\prime}$ such that

$$
|f(x)| \leq c^{\prime}(1+|x|) \quad \text { and } \quad|g(x)| \leq c^{\prime}(1+|x|) \quad \forall x \in \mathbb{R} .
$$

We have the following theorem:

TheOREM 1.1. Given $\left(u_{0}, u_{1}\right) \in V \times H$ arbitrarily, the problem (1.3)(1.6) has a unique solution (defined in a suitable weak sense) satisfying

$$
u \in C\left(\mathbb{R}^{+} ; V\right) \cap C^{1}\left(\mathbb{R}^{+} ; H\right) .
$$

Moreover, its energy is nonincreasing. If $\left(u_{0}, u_{1}\right) \in W \times V$ is such that

$$
f\left(u_{1}\right) \in H \quad \text { and } \quad \partial_{\nu} u_{0}+(m . \nu)\left(a u_{0}+g\left(u_{1}\right)\right)=0 \quad \text { on } \Gamma_{1} \text {, }
$$

then the solution (called a strong solution) of (1.3)-(1.6) has the following regularity properties:

$$
\begin{gathered}
u \in L^{\infty}\left(\mathbb{R}^{+} ; W\right), \\
u^{\prime} \in L^{\infty}\left(\mathbb{R}^{+} ; V\right), \\
u^{\prime \prime}, f\left(u^{\prime}\right) \in L^{\infty}\left(\mathbb{R}^{+} ; H\right) .
\end{gathered}
$$

In the case where $f=0$ or $g=0$, the system (1.3)-(1.6) was studied earlier for example by Komornik [5, 6], Nakao [9] and Zuazua [11]. Decay results for the solutions of (1.3)-(1.6) were obtained in this case. The aim of this paper is to extend these results to the case of nonlinear internal and boundary feedbacks. Moreover, we obtain rather precise decay estimates of the energy under suitable growth assumptions on $f, g$ and a particular choice of the constant $a$. We give a better result than the one found in Komornik [7] when the dimension of the space is equal to 2 .

Assume in the sequel that

$$
\text { either } \quad \Gamma_{0} \neq \emptyset \quad \text { or } \quad a>0 \text { and } \inf _{\Gamma_{1}}(m . \nu)>0
$$


and that

$$
\bar{\Gamma}_{0} \cap \bar{\Gamma}_{1}=\emptyset \quad \text { if } n>3 .
$$

We prove the following result.

Theorem 1.2. Assume (1.2), (1.15), (1.16) and assume that there exist two positive constants $c_{1}, c_{2}$ such that

$$
\begin{aligned}
c_{1}|x| & \leq|g(x)| \leq c_{2}|x|, \quad \forall x \in \mathbb{R}, \\
\text { (1.18) } \quad \frac{1}{2}\left(3 a R^{2}-n\right) c_{1}|x| & \leq|f(x)| \leq \frac{1}{2}\left(3 a R^{2}-n\right) c_{2}|x|, \quad \forall x \in \mathbb{R},
\end{aligned}
$$

and

$$
\frac{1}{R^{2}} \max \left\{n-2, \frac{n}{3}\right\} \leq a<\frac{n}{R^{2}}
$$

Then for every $\left(u_{0}, u_{1}\right) \in V \times H$ the solution of (1.3)-(1.6) satisfies the energy estimate

$$
E(t) \leq E(0) e^{1-\omega t}, \quad \forall t \geq 0,
$$

where $\left(2 R+R^{2} c_{2}+1 / c_{1}\right) \omega=n-a R^{2}$.

REMARKs. 1. We note that Theorem 1.2 remains valid without the assumption (1.16). However, the proof becomes more delicate because the strong solutions are not sufficiently smooth to justify the computations given below. One can overcome this difficulty by applying a domain approximation argument. (See Komornik and Zuazua [8] for a similar study of the wave equation).

2. By hypothesis (1.15) the expression

$$
\|u\|_{V}^{2}=\int_{\Omega}|\nabla u|^{2} d x+a \int_{\Gamma_{1}}(m . \nu) u^{2} d \Gamma
$$

defines a norm on $V$, which is equivalent to the norm induced by $H^{1}(\Omega)$; consequently, $V$ is a Hilbert space with this norm.

3. If we choose $a=\left(1 / R^{2}\right) \max \{n-2, n / 3\}$ and $c_{1}=c_{2}=1 / R$ (i.e. $f=0$ if $n=1,2,3$ and $g(x)=(1 / R) x$ for $x \in \mathbb{R})$, then we find that the solutions of (1.3)-(1.6) satisfy estimates (1.20) with $\omega=n /(6 R)$ if $n=1,2,3$ and $\omega=1 /(2 R)$ if $n>3$. This gives a better result than the one found in Komornik [7] when the dimension of the space is equal to 2 .

4. The decay estimates will be obtained by applying a Lyapunov type method based on an integral inequality applied earlier in Komornik $[4,5,6]$ and Zuazua [11].

For the proof of Theorem 1.2, we use the following lemma. 
Lemma 1.3 (Komornik [4]). Let $E: \mathbb{R}^{+} \rightarrow \mathbb{R}^{+}$be a nonincreasing function and assume that there are two constants $\alpha \geq 0$ and $T>0$ such that

$$
\int_{t}^{\infty} E(s)^{\alpha+1} d s \leq T E(0)^{\alpha} E(t), \quad \forall t \in \mathbb{R}^{+} .
$$

Then

$$
E(t) \leq E(0)\left(\frac{T+\alpha t}{T+\alpha T}\right)^{-1 / \alpha}, \quad \forall t \geq 0, \text { if } \alpha>0
$$

and

$$
E(t) \leq E(0) e^{1-t / T}, \quad \forall t \geq 0, \text { if } \alpha=0 .
$$

2. Proof of Theorem 1.2. We note that it is sufficient to prove the estimate (1.20) for strong solutions: the general case then follows by an easy density argument as in Zuazua [11]. We henceforth assume that $u$ is a strong solution of (1.3)-(1.6).

We begin by establishing two identities. They will be obtained by multiplying the equation (1.3) with $u^{\prime}$ and $2 m . \nabla u+2 a R^{2} u$, respectively, and by integrating by parts in $\Omega \times(S, T)$ where $(S, T)$ is an arbitrarily fixed bounded interval in $\mathbb{R}^{+}$.

Lemma 2.1. The function $E: \mathbb{R}^{+} \rightarrow \mathbb{R}^{+}$is nonincreasing, locally absolutely continuous and

$$
E^{\prime}(t)=-\int_{\Omega} u^{\prime} f\left(u^{\prime}\right) d x-\int_{\Gamma_{1}}(m . \nu) u^{\prime} g\left(u^{\prime}\right) d \Gamma \quad \text { a.e. in } \mathbb{R}^{+} .
$$

Pr o of. Fixing $0 \leq S<T<\infty$ arbitrarily, we have the equality

$$
\begin{aligned}
0= & \int_{S}^{T} \int_{\Omega} u^{\prime}\left(u^{\prime \prime}-\Delta u+f\left(u^{\prime}\right)\right) d x d t \\
= & \int_{S}^{T} \int_{\Omega}\left(u^{\prime} u^{\prime \prime}+\nabla u \cdot \nabla u^{\prime}+u^{\prime} f\left(u^{\prime}\right)\right) d x d t-\int_{S}^{T} \int_{\Gamma} u^{\prime} \partial_{\nu} u d \Gamma d t \\
= & \int_{S}^{T} \int_{\Omega}\left(u^{\prime} u^{\prime \prime}+\nabla u \cdot \nabla u^{\prime}+u^{\prime} f\left(u^{\prime}\right)\right) d x d t \\
& +\int_{S}^{T} \int_{\Gamma_{1}}(m . \nu)\left(a u+g\left(u^{\prime}\right)\right) u^{\prime} d \Gamma d t,
\end{aligned}
$$

whence

$$
E(S)-E(T)=\int_{S}^{T} \int_{\Omega} u^{\prime} f\left(u^{\prime}\right) d x d t+\int_{S}^{T} \int_{\Gamma_{1}}(m . \nu) u^{\prime} g\left(u^{\prime}\right) d \Gamma d t .
$$


Since $m . \nu \geq 0$ on $\Gamma_{1}$ and since $x f(x), x g(x) \geq 0$ for $x \in \mathbb{R}$, the right hand side of (2.2) is nonnegative; hence $E$ is nonincreasing. It also follows from (2.2) that $E$ is locally absolutely continuous and that (2.1) is satisfied.

Lemma 2.2. Putting for brevity

$$
M=2 m . \nabla u+2 a R^{2} u,
$$

for all $0 \leq S<T<\infty$ we have

$$
\begin{aligned}
\left(2 a R^{2}\right. & +2-n) \int_{S}^{T} \int_{\Omega}|\nabla u|^{2} d x d t+\left(n-2 a R^{2}\right) \int_{S}^{T} \int_{\Omega}\left(u^{\prime}\right)^{2} d x d t \\
= & -\left[\int_{\Omega} M u^{\prime} d x\right]_{S}^{T}-\int_{S} \int_{\Omega} M f\left(u^{\prime}\right) d x d t+\int_{S}^{T} \int_{\Gamma_{0}}(m . \nu)\left(\partial_{\nu} u\right)^{2} d \Gamma d t \\
& +\int_{S}^{T} \int_{\Gamma_{1}}(m . \nu)\left(\left(u^{\prime}\right)^{2}-|\nabla u|^{2}-M\left(a u+g\left(u^{\prime}\right)\right)\right) d \Gamma d t .
\end{aligned}
$$

Proof. We have

$$
\begin{aligned}
0 & =\int_{S}^{T} \int_{\Omega} M\left(u^{\prime \prime}-\Delta u+f\left(u^{\prime}\right)\right) d x d t \\
& =\left[\int_{\Omega} u^{\prime} M d x\right]_{S}^{T}+\int_{S}^{T} \int_{\Omega} M f\left(u^{\prime}\right) d x d t-\int_{S}^{T} \int_{\Omega}\left(u^{\prime} M^{\prime}+M \Delta u\right) d x d t .
\end{aligned}
$$

Integrating by parts and using the relation $\operatorname{div} m=n$ we transform the inner integral in the last term as follows:

$$
\begin{aligned}
\int_{\Omega}\left(u^{\prime} M^{\prime}+\right. & M \Delta u) d x \\
= & \int_{\Omega}\left(m . \nabla\left(u^{\prime}\right)^{2}+2 a R^{2}\left(u^{\prime}\right)^{2}-\nabla u \cdot \nabla M\right) d x+\int_{\Gamma} M \partial_{\nu} u d \Gamma \\
= & \int_{\Omega}\left(m . \nabla\left(u^{\prime}\right)^{2}+2 a R^{2}\left(u^{\prime}\right)^{2}-2|\nabla u|^{2}-m \cdot \nabla|\nabla u|^{2}-2 a R^{2}|\nabla u|^{2}\right) d x \\
& +\int_{\Gamma} M \partial_{\nu} u d \Gamma \\
= & -\left(2 a R^{2}+2-n\right) \int_{\Omega}|\nabla u|^{2} d x-\left(n-2 a R^{2}\right) \int_{\Omega}\left(u^{\prime}\right)^{2} d x \\
& +\int_{\Gamma_{0}}\left(-(m . \nu)|\nabla u|^{2}+(2 m . \nabla u) \partial_{\nu} u\right) d \Gamma \\
& +\int_{\Gamma_{1}}(m . \nu)\left(\left(u^{\prime}\right)^{2}-|\nabla u|^{2}-M\left(a u+g\left(u^{\prime}\right)\right)\right) d \Gamma .
\end{aligned}
$$


Substituting this equality into (2.5) and using the equality $\nabla u=\nu \partial_{\nu} u$ on $\Gamma_{0}$, we obtain the identity $(2.4)$.

Lemma 2.3. The following estimates hold true:

$$
\left|\int_{\Omega} M u^{\prime} d x\right| \leq 2 R E(t)
$$

and for all $\varepsilon>0$

$$
\left|\int_{\Omega} M f\left(u^{\prime}\right) d x\right| \leq \varepsilon \int_{\Omega} f^{2}\left(u^{\prime}\right) d x+\frac{R^{2}}{\varepsilon}\left(\int_{\Omega}|\nabla u|^{2} d x+a \int_{\Gamma_{1}}(m . \nu) u^{2} d \Gamma\right) .
$$

Pr o of. Using (1.19) we have

$$
\begin{array}{rl}
\int_{\Omega}\left|2 m . \nabla u+2 a R^{2} u\right|^{2} d & x-\int_{\Omega}|2 m . \nabla u|^{2} d x \\
& =\int_{\Omega}\left(4 a^{2} R^{4} u^{2}+8 a R^{2}(m . \nabla u) u\right) d x \\
& =4 a R^{2} \int_{\Gamma_{1}}(m \cdot \nu) u^{2} d \Gamma+4 a R^{2}\left(a R^{2}-n\right) \int_{\Omega} u^{2} d x \\
& \leq 4 a R^{2} \int_{\Gamma_{1}}(m \cdot \nu) u^{2} d \Gamma .
\end{array}
$$

Hence, using (1.7) we conclude that

$$
\begin{aligned}
\left|\int_{\Omega} M u^{\prime} d x\right| & \leq \frac{1}{4 R} \int_{\Omega}\left|2 m . \nabla u+2 a R^{2} u\right|^{2} d x+R \int_{\Omega}\left(u^{\prime}\right)^{2} d x \\
& \leq \frac{1}{4 R} \int_{\Omega}|2 m . \nabla u|^{2} d x+a R \int_{\Gamma_{1}}(m \cdot \nu) u^{2} d \Gamma+R \int_{\Omega}\left(u^{\prime}\right)^{2} d x \\
& \leq R\left(\int_{\Omega}\left(\left(u^{\prime}\right)^{2}+|\nabla u|^{2}\right) d x+a \int_{\Gamma_{1}}(m . \nu) u^{2} d \Gamma\right)=2 R E(t)
\end{aligned}
$$

and hence (2.6) follows.

Next by a similar computation, for all $\varepsilon>0$, we obtain

$$
\begin{aligned}
\left|\int_{\Omega} M f\left(u^{\prime}\right) d x\right| & \leq \varepsilon \int_{\Omega} f\left(u^{\prime}\right)^{2} d x+\frac{1}{4 \varepsilon} \int_{\Omega}|M|^{2} d x \\
& \leq \varepsilon \int_{\Omega} f\left(u^{\prime}\right)^{2} d x+\frac{1}{4 \varepsilon}\left(\int_{\Omega}|2 m . \nabla u|^{2} d x+4 a R^{2} \int_{\Gamma_{1}}(m . \nu) u^{2} d \Gamma\right) \\
& \leq \varepsilon \int_{\Omega} f\left(u^{\prime}\right)^{2} d x+\frac{R^{2}}{\varepsilon}\left(\int_{\Omega}|\nabla u|^{2} d x+a \int_{\Gamma_{1}}(m . \nu) u^{2} d x\right),
\end{aligned}
$$

which is (2.7), and the lemma follows. 
Lemma 2.4. On $\Gamma_{1}$ the following estimate holds true:

$$
-M\left(a u+g\left(u^{\prime}\right)\right)-|\nabla u|^{2} \leq R^{2} g\left(u^{\prime}\right)^{2}-a^{2} R^{2} u^{2} .
$$

Proof. We have

$$
\begin{aligned}
-M\left(a u+g\left(u^{\prime}\right)\right) \leq & 2|m . \nabla u| \cdot\left|a u+g\left(u^{\prime}\right)\right|-2 a^{2} R^{2} u^{2}-2 a R^{2} u g\left(u^{\prime}\right) \\
\leq & |\nabla u|^{2}+R^{2}\left(a^{2} u^{2}+g\left(u^{\prime}\right)^{2}+2 a u g\left(u^{\prime}\right)\right) \\
& -2 a^{2} R^{2} u^{2}-2 a R^{2} u g\left(u^{\prime}\right) \\
\leq & |\nabla u|^{2}-a^{2} R^{2} u^{2}+R^{2} g\left(u^{\prime}\right)^{2} .
\end{aligned}
$$

The lemma follows.

Let us return to the identity (2.4). Applying the preceding two lemmas and using also the assumptions (1.2), (1.17), (1.19) and the definition (1.7) of energy, we deduce easily from this identity the following inequality for all $0 \leq S<T<\infty$ and all $\varepsilon>0$ :

$$
\begin{aligned}
2 R^{2}\left(a-\frac{1}{\varepsilon}\right) \int_{S}^{T} E(t) d t \leq & 2 R(E(S)+E(T))+\varepsilon \int_{S}^{T} \int_{\Omega} f\left(u^{\prime}\right)^{2} d x d t \\
& +\left(\frac{1}{c_{1}}+R^{2} c_{2}\right) \int_{S}^{T} \int_{\Gamma_{1}}(m . \nu) u^{\prime} g\left(u^{\prime}\right) d \Gamma d t \\
& +\left(3 a R^{2}-n-\frac{R^{2}}{\varepsilon}\right) \int_{S}^{T} \int_{\Omega}\left(u^{\prime}\right)^{2} d x d t
\end{aligned}
$$

Choosing $\varepsilon=2 R^{2} /\left(3 a R^{2}-n\right)$, using the assumption (1.18) and the identity (2.2), we conclude from (2.9) that (in the case where $n=3 a R^{2}$, we have, by (1.18), $f=0$; then we let $\varepsilon \rightarrow \infty$ in (2.9))

$$
\begin{aligned}
\left(n-a R^{2}\right) \int_{S}^{T} E(t) d t \leq & \left(2 R+R^{2} c_{2}+\frac{1}{c_{1}}\right) E(S) \\
& +\left(2 R-R^{2} c_{2}-\frac{1}{c_{1}}\right) E(T) .
\end{aligned}
$$

Since (cf. (1.17))

$$
2 R-R^{2} c_{2}-\frac{1}{c_{1}} \leq 2 R-R^{2} c_{1}-\frac{1}{c_{1}}=-\left(R \sqrt{c_{1}}-\frac{1}{\sqrt{c_{1}}}\right)^{2} \leq 0,
$$

letting $T \rightarrow \infty$ we conclude that

$$
\int_{S}^{\infty} E(t) d t \leq \frac{1}{n-a R^{2}}\left(2 R+R^{2} c_{2}+\frac{1}{c_{1}}\right) E(S), \quad \forall S \geq 0,
$$

and we may then complete the proof by applying Lemma 1.3. 


\section{References}

[1] J. B. Ball, On the asymptotic behavior of generalized processes with applications to nonlinear evolution equations, J. Differential Equations 27 (1978), 224-265.

[2] F. Conrad and M. Pierre, Stabilization of second order evolution equations by unbounded nonlinear feedback, Ann. Inst. Henri Poincaré 11 (1994), 485-515.

[3] A. Guesmia, Stabilisation frontière non linéaire d'un système isotropique d'élasticité, submitted.

[4] V. Komornik, Exact Controllability and Stabilization, the Multiplier Method, Masson-Wiley, Paris, 1994.

[5] - Decay estimates for the wave equation with internal damping, in: Proc. Conf. Control Theory, Vorau 1993, Internat. Ser. Numer Anal. 118, Birkhäuser, Basel, 1994, 253-266.

[6] - On the nonlinear boundary stabilization of the wave equation, Chinese Ann. Math. Ser. B 14 (1993), 153-164.

[7] - Rapid boundary stabilization of the wave equation, SIAM J. Control Optim. 29 (1991), 197-208.

[8] V. Komornik and E. Zuazua, A direct method for the boundary stabilization of the wave equation, J. Math. Pures Appl. 69 (1990), 33-54.

[9] M. Nakao, On the decay of solutions of some nonlinear dissipative wave equations in higher dimensions, Math. Z. 193 (1986), 227-234.

[10] - Energy decay for the wave equation with a nonlinear weak dissipation, Differential Integral Equations 8 (1995), 681-688.

[11] E. Zuazua, Uniform stabilization of the wave equation by nonlinear boundary feedback, SIAM J. Control Optim. 28 (1990), 446-477.

Institut de recherche mathématique avancée

Université Louis Pasteur et CNRS

7 , rue René Descartes

67084 Strasbourg Cedex, France

E-mail: guesmia@math.u-strasbg.fr 